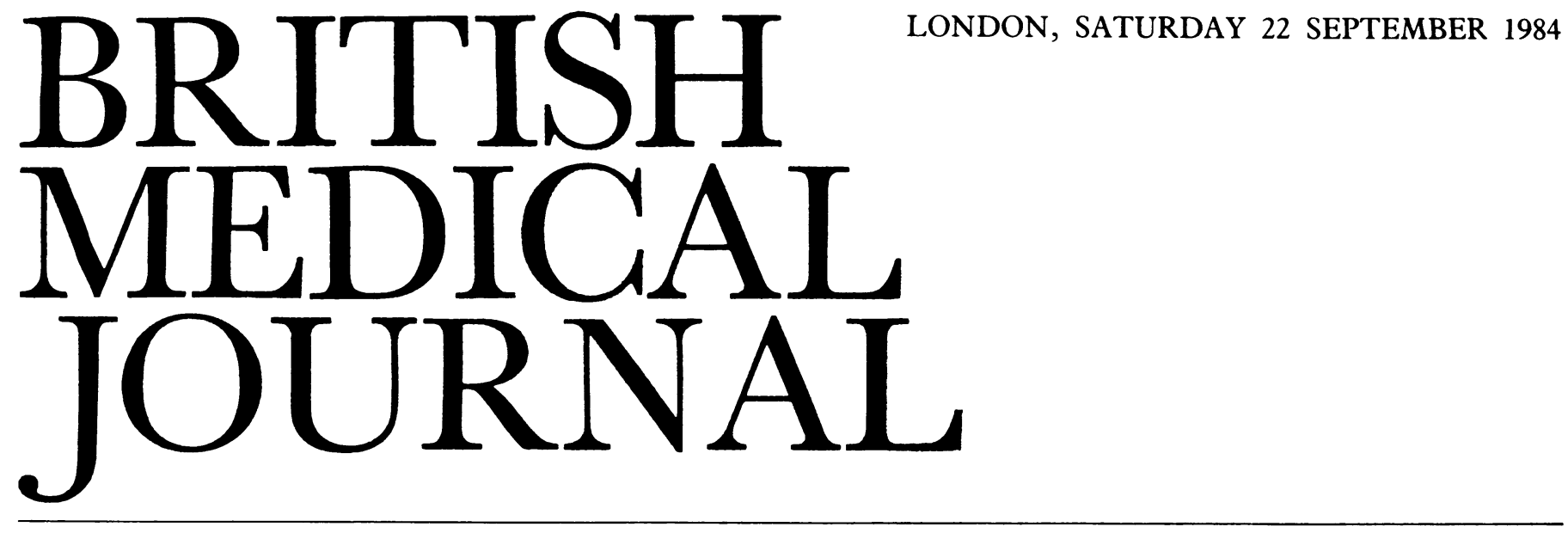

\title{
Does moderate drinking cause mental impairment?
}

A maximum safe level of alcohol consumption for men is between six and seven units of alcohol a day $(60 \mathrm{~g}$ pure ethanol) and for women about half that amount ( 1 unit $=9 \mathrm{~g}$ pure ethanol=one single measure of spirits, half a pint of beer, or one glass of wine).' Yet, if we accept the conclusions of several recent studies, men and women drinking well within these limits are likely to show some enduring mental impairment even when they have no alcohol in their blood. Is this evidence valid?

Clinically defined alcoholics show a high incidence of cognitive impairment, ${ }^{2-4}$ and Elizabeth Parker has argued that the effects of alcohol on cognitive function represent a continuum between light social drinking at one end of the spectrum, through heavy drinking, to Korsakoff's syndrome at the other extreme. ${ }^{5}$ Most of the evidence for such a continuum of effects comes from correlational studies, with all their attendant pitfalls. Seven studies have found significant, if small, correlations between certain indices of alcohol consumption and various measures of cognitive function among moderate drinkers. ${ }^{6-12}$ Most commonly, problem solving and abstraction abilities, memory, and psychomotor abilities have shown correlations with various measures of alcohol consumption. ${ }^{6-11} \mathrm{~A}$ common thread running through all these seven studies is that some measure of mental function has been shown to be negatively correlated with some index of alcohol consumption, though in the study by Hannon et al heavier male drinkers performed better in one test (Trails B) than did their lighter drinking counterparts. ${ }^{12}$

One other common theme running through the research is the curious finding that more intelligent people (as measured by vocabulary, an index largely unaffected by alcohol) tend to drink less. This might explain many of the correlations between alcohol consumption and cognitive abilities; a third variable-namely, intelligence-may influence both. Only in the report by Jones and Jones were heavier and light drinking groups matched for intelligence, and their study showed that one of three indices of verbal memory was significantly lower among the heavy drinkers. ${ }^{9}$ If these results were replicated, then such correlational evidence as we already have would have to be taken more seriously. Many other variables could explain the apparent link between cognition and alcohol consumption, including social class, diet, history of head injury, genetic predisposition, depression, and anxiety. In none of the studies have most of these variables been controlled for, nor has there been control for the possibility that heavier drinkers may have been more likely to have drunk alcohol shortly before they were tested.

Given the pitfalls of correlational research, then, we must depend on longitudinal investigations to find an answer. Unfortunately, there has been very little such research. A study in Australia by Cala et al followed up 26 light to moderate drinkers who agreed to abstain for six months. ${ }^{13} 14$ The mean increases in verbal intelligence quotient, performance intelligence quotient, and memory quotient were 5,8 , and 6 respectively. Such increases, however, are all well within the changes attributable to a practice effect, ${ }^{15} 16$ whatever Cala et al have argued. ${ }^{17}$ Furthermore, these changes can be interpreted only by comparing them with an adequate control group of people who did not abstain, and Cala et al did not do this. Their claim that, on psychometric measures, $70 \%$ of light to moderate drinkers in their sample had brain damage on recruitment to their trial $^{14}$ was based on a whimsical set of psychometric criteria for brain damage, one of which was that the memory quotient should be over 15 points below their verbal intelligence quotient. ${ }^{13} \mathrm{Dr}$ Cala has recently reported that 12 of the 26 people who were retested a further six months later, having resumed drinking at a mean rate of about five units a day, maintained the improvements said to have been attributable to an earlier six months' abstinence ( $L$ Cala, personal communication, 1984). That these improvements may not have been due to abstinence but to a practice effect is suggested by the fact that some of those who showed improvement had been drinking very little on entry (two to three units a day).

The only other longitudinal study followed up 62 problem drinkers over three weeks. ${ }^{18}$ Some of these people abstained, some of them drank just over one unit a day, while the remainder drank an average of about five units a day. Only the improvements of the abstainers and the light drinkers was statistically significant, in the only test given-a measure of psychomotor speed and coordination (the WAIS digit-symbol test). The heavy drinking group were older than the other two groups, however, and had been heavier drinkers in the past. Furthermore, the authors reported no period of abstinence before testing, and so possibly their performance was affected by the effects of the previous evening's intake. 
Hence we still have no good evidence to suggest that we need to revise the safe daily drinking limits of $60 \mathrm{~g}$ ethanol for men and $30 \mathrm{~g}$ for women, though further longitudinal research is required to test some of the suggestive findings from correlational research. Until then the conclusions of MacVane et al and Parsons and Fabian must stand: "It would seem advisable that additional carefully controlled studies of social drinking be completed before any pronouncement concerning the dangers of social drinking are disseminated to the public. ${ }_{810}$

IAN ROBERTSON

Principal Clinical Psychologist,

Astley Ainslie Hospital.

Edinburgh EH9 2HL

I Paton A, Saunders JB. ABC of alcohol. Definitions. Br Med f 1981:283:1248-50

Parsons OA. Neuropsychological deficits in alcoholics: facts and fancies. Alcoholesm: Clintcal and Experimental Research 1977:1:51-6.

Goodwin DW, Hill SY. Chronic effects of alcohol and other psychoactive drugs on intellect. learning and memory. In: Rankin JG, ed. Alcohol, drugs and brain damage. Toronto: Addiction

Research Foundation, $1975: 55-70$.
Ron MA. Brain damage in chronic alcoholism: a neuropathological, neuroradiological and psychological review. Psychol Med 1977;7:103-12

In: W' Parker DA. Towards an epidemiology of cognitive deficits among alcohol consumers In: Wilkinson DA, ed. Cerebral deficits in alcoholism. Toronto: Addiction Research
Foundation, 1983:21-46. Parter ES Noble EP Alcohol

Alcohol 1980;38:1224-32.

Parker ES, Birnbaum IM, Boyd R, Noble EP. Neuropsychological decrements as a function of alcohol intake in male students. Alcoholism: Clinical and Experimental Research 1980:4 $1330-4$ MacVane J, Butters N, Montgomery K, Farber J. Cognitive functioning in men social drinkers a replication study. F Stud Alcohol 1982;43:81-95.

Jones MK, Jones BM. The relationship of age and drinking history to the effects of alcohol on memory in women. I Stud Alcohol 1980;41:179-86.

Parsons OA, Fabian MS. Comments on cognitive functioning in men social drinkers: a replication study. F Stud Alcohol 1982;43:178-82

Parker DA, Parker ES, Brody JA, Schoenberg R. Alcohoi use and cognitive loss among employed men and women. Am f Public Health 1983;73:521-6.

Hannon R, Dav CL, Butler AM, Larson AJ, Casey M. Alcohol consumption and cognitive functioning in college students. I Stud Alcohol 1983;44:283-98.

Canes L, Burns P, Davis R, Stenhouse N, Mastaglia F. Results of computerized tomography, psychometric testing and dietary studies in social drinkers, with emphasis on

Cala L, Burns P, Davis R, Jones B, Stenhouse N. Alcohol and brain damage. Australlan Alcohol Drug Revier: 1984:3:53-8.

Matarazzo JD, Carmody JP, Jacobs LD. Test-retest reliability and stability of the W'AIS: a literature review and implications for clinical practice. $\mathcal{F}$ Clin Neuropsychol 1980;2:89-105.

6 Bowden SC, Walsh KW. Social drinking and cognitive impairment. Med $\mathscr{f}$ A ust 1984:140: 175-6. Cala L, Jones B, Burns P, Davis R, Stenhouse N, Mastaglia F. Reply to Bowden and Walsh. Med F Aust 1984;140:176.

8 Wilkinson DA, Sanchez-Craig M. Relevance of brain dysfunction to treatment objectives: should alcohol-related cognitive deficits influence the way we think about treatment? Addic Behav 1981;6:253-60

\section{Congenital cytomegalovirus infection: a dilemma}

Congenital cytomegalovirus infection is going to be much more difficult to prevent than congenital rubella-and that is not proving easy, cheap, or quick to achieve. The problem is substantial because, except in years when there is an epidemic of rubella, cytomegalovirus infection in pregnancy is considerably more common.

The first difficulty is that, unlike rubella, which can usually be detected clinically, cytomegalovirus infection in adults is almost always symptomless-and so must be diagnosed by virological tests. Even in infants infected congenitally many of the infections are silent and are detected only in the laboratory. Around $40 \%$ to $45 \%$ of women in Britain have no antibody to cytomegalovirus and are therefore non-immune. ${ }^{1-4}$ Various studies have shown that just under $1 \%$ of these seronegative women have a primary infection in pregnancy, ${ }^{256}$ and some of these-variously reported as a fifth to a half-will transmit the virus to their child. ${ }^{15-8}$ Routine virological testing has shown that overall in Britain about $0.3 \%$ to $0.4 \%$ of infants are congenitally infected with cytomegalovirus (although recurrent as well as primary infection will contribute to this) ${ }^{4}$

Not all fetal infections result in permanent damage, and fortunately the classic syndrome of severe cytomegalic inclusion disease is rare. ${ }^{9}$ Nevertheless, about a fifth of infected infants are damaged, with signs of generalised infection and lesions in the central nervous system. ${ }^{6}$ Most survive, but some are left with mental impairment or deafness. ${ }^{1011}$ Even in infants who appear normal at birth mental retardation, and more commonly deafness, may become manifest later in life. ${ }^{610} 11$ The prognosis is, perhaps not surprisingly, better in asymptomatic infants than in those with the signs and stigmata of infection at birth..$^{610^{12}}$ In one American study children with asymptomatic congenital infection were re-examined together with a control group at the ages of $31 / 2$ to 7 years. ${ }^{13}$ The infected children had lower intelligence quotients and a higher rate of "school failure" and of bilateral hearing loss. To make matters more difficult, some infants with symptoms at birth develop normally, so that hard and fast rules cannot be laid down for calculating the prognosis in an individual child.

It might be expected that, as with rubella, fetal damage from cytomegalovirus would be both more common and more severe if the infection is contracted in the earlier stages of pregnancy. Too few cases have been reported in various surveys to allow definite conclusions to be drawn. What is certain, however, is that maternal infection in the second and third trimester ${ }^{410}$ may be followed not only by spread of virus to the fetus but by fetal damage with sequelae. ${ }^{4514}$ Griffiths and Baboonian found that transplacental infection was (rather surprisingly) more common in the third than in the first trimester. ${ }^{5}$ Unlike other workers they detected no transmission of virus in the second trimester but found excess fetal loss from abortion and stillbirth in pregnancies infected at this stage. There was no virological proof that cytomegalovirus had been responsible, but the suspicion remains.

Cytomegalovirus is a herpesvirus and, like other members of that family, shows the property of latency. After primary infection the virus becomes latent (probably in white blood cells) and may later reactivate to cause recurrent infection. Unfortunately (and contrary to earlier reports) congenital infection may follow recurrent maternal infection. ${ }^{46} 15-17$ Recurrences-presumably due to reactivation of latent virus-are not uncommon in pregnancy. ${ }^{6}$ Infants infected as a result of recurrent infection are usually symptomless, ${ }^{671516}$ but generalised infection and neurological damage have been reported in a few cases. ${ }^{471017}$ Clearly, therefore, maternal antibody does not prevent infection of the fetus and reactivated maternal virus may occasionally damage it. These observations probably rule out the use of vaccine. Effective cytomegalovirus vaccines have been developed, but they contain live attenuated virus, which may well become latent after vaccination-a point that will be very difficult to disprove. ${ }^{1819}$

What, then, can be done to minimise or preferably prevent fetal damage by cytomegalovirus? We have no obvious answer from current knowledge. The virus causes damage both before and after the time at which pregnancy could be terminated, so not all women diagnosed as infected could be offered this. Diagnosis itself is not easy and would require expensive tests in a nationwide screening programme. And even in those cases detected in early pregnancy termination would result in the loss of some babies either not infected or, even if infected, destined to develop normally.

Three groups-Peckham and her coworkers ${ }^{4}$ and Griftiths and Baboonian ${ }^{5}$ in Britain, and Ahlfors and colleagues in Sweden ${ }^{717}$-have recently concluded that routine screening of pregnant women for evidence of primary cytomegalovirus infection is not helpful. Termination does not seem to be an 